\title{
Pratiques historiennes d'une génération : Henri Beyle et ses pairs
}

Historian Practices of a Generation: Henri Beyle and His Peers

\section{Aurélien Lignereux}

\section{(2) OpenEdition \\ Journals}

Édition électronique

URL : http://journals.openedition.org/recherchestravaux/892

ISSN : 1969-6434

\section{Éditeur}

UGA Éditions/Université Grenoble Alpes

\section{Édition imprimée}

ISBN : 978-2-37747-006-8

ISSN : 0151-1874

\section{Référence électronique}

Aurélien Lignereux, «Pratiques historiennes d'une génération : Henri Beyle et ses pairs », Recherches \& Travaux [En ligne], 90 | 2017, mis en ligne le 15 juin 2017, consulté le 08 septembre 2020. URL : http:// journals.openedition.org/recherchestravaux/892

Ce document a été généré automatiquement le 8 septembre 2020

(C) Recherches \& Travaux 


\title{
Pratiques historiennes d'une génération : Henri Beyle et ses pairs
}

\author{
Historian Practices of a Generation: Henri Beyle and His Peers
}

Aurélien Lignereux

1 En avertissant en tête de sa Vie de Napoléon que «L'abrégé qui suit n'est pas une histoire proprement dite, c'est l'histoire pour les contemporains témoins des faits ${ }^{1}$ », Stendhal dévoilait d'emblée la nature à la fois ambiguë et ambitieuse de son projet historien : il écrit d'abord pour ou plutôt contre ses contemporains ayant la mémoire courte, mais bien conscient de ne livrer qu'un état provisoire des savoirs, en attendant la publication à venir des Mémoires de témoins de premier plan. Voilà qui laisse pressentir les difficultés d'appréhension d'une œuvre ébauchée en deux tentatives successives (Vie de Napoléon, 1817-1818 ; Mémoires sur Napoléon, 1836-1837), d’un travail à la fois sincère et plagiaire, et dont l'autorité que construisent les seuils préfaciers joue de l'ambivalence entre le rédacteur-compilateur qu'il entend être et l'observateur direct qu'il dit avoir été. Autant dire que ces essais sont de nature à brouiller le partage qu'il est usage d'opérer entre histoire et Mémoires, car c'est en partie la distance chronologique qui fait considérer ces textes comme relevant de l'une ou des autres, au gré d'effets de lecture anticipés par Stendhal. Puisque Napoléon est au centre de ces œuvres, se surimpose le questionnement spécifique au genre de la biographie, soit la mise à nu d'un caractère au fil d'un portrait en situation et en contexte, à cheval entre l'histoire et la littérature, ou plutôt rétrospectivement à cheval tant cette grande séparation est postérieure ${ }^{2}$.

2 Ces œuvres inabouties de Stendhal se situent donc quelque part dans le triangle formé par l'histoire, les Mémoires et la biographie, qu'unit une même ambition de dire vrai. Dans ces conditions, que nous revient-il de faire? Soupeser le geste de Stendhal qui entreprend à sa façon d'" écrire la mémoire ${ }^{3}$ ", relever ce qui rattache ces textes à l'histoire, apprécier le travail du style qui esthétise d'ores et déjà ces esquisses ? Pareil effort s'annonce vain si bien que plutôt de rechercher un Stendhal historien ou un Stendhal mémorialiste, on pourrait être tenté d'y retrouver Stendhal tout court. Or plutôt qu'un tel tête-à-tête, nous procéderons au mouvement inverse, en cherchant 
d'autres historiens en quête d'eux-mêmes durant cette période. En effet, les interrogations précédentes valent en vérité pour bien des individus prenant alors la plume pour rendre compte de ce qu'ils ont vécu, pour penser cette expérience historique et la faire partager, tout en tâtonnant d'un registre d'écriture à l'autre. Une telle démarche est même si courante qu'il faut parer en retour un risque de dilution : la comparaison va se resserrer sur quelques individus, qu'il serait certes bien maladroit de traiter en alter-ego de Stendhal - égotisme oblige -, mais qu'il est légitime de considérer comme ses pairs en tant que collègues, conscrits ou compatriotes.

Ils sont nés autour de 1780 ; hauts fonctionnaires, ils ont servi Napoléon dans l'Europe conquise, puis ont pris leurs distances avec l'empereur au moment de sa chute avant de suivre des parcours contrastés. Plusieurs ont connu une carrière préfectorale, quelques-uns sont passés ministres; Henri Beyle est devenu Stendhal. Tous cependant ont écrit des Mémoires, des Souvenirs ou tout autre livre à caractère historique. Les formes sont diverses, les genres hésitants ; quelques œuvres ont été publiées de leur vivant, d'autres à titre posthume; certaines sont restées à l'état d'archives. Et c'est précisément cette variété qui est susceptible d'éclairer sous un jour nouveau les tentatives de Stendhal, car ses incursions en histoire ont été aussi celles de ses pareils. Mettre en parallèle les écrits historiques de Stendhal avec ceux de ses pairs, c'est donc dégager une aspiration commune, c'est se donner les moyens de comprendre l'individu par le groupe auquel il a pu appartenir, c'est relativiser aussi le cas Stendhal en se souvenant à la fois qu'il fut un auditeur au Conseil d'État et que nombre de ses collègues furent également des auteurs. Mais moins qu'une approche sociologique rigide dont on sait qu'elle achoppera sur le beylisme, c'est davantage une hypothèse socio-historique qui sera mise en œuvre, en termes d'opportunités professionnelles, de choix de vie et finalement d'écritures possibles.

\section{Ni témoin ni collègue : le Stendhal des historiens de l'Empire}

4 Encore faut-il évaluer au préalable les usages que les historiens réservent à Stendhal, ce qui revient plus largement à son inscription dans les groupes sociaux. Alors que dans les travaux sur les monarchies censitaires, Stendhal sert fréquemment d'introducteur pour pénétrer, par ses romans, dans les salons de province ou pour entrevoir le dessous de cartes préfectoral, les études napoléoniennes ne lui accordent que peu de place. Il est vrai que s'intéresser à la vie d'Henri Beyle amène dans un terrain déjà occupé par les stendhaliens. Et pour cause : reconstituer son itinéraire suppose une véritable spécialisation tant cela exige une familiarité fine avec ses manuscrits, une collecte patiente des autographes, la maitrise du Fichier stendhalien ${ }^{4}$. Tout un travail de recoupements s'impose pour saisir Stendhal à travers ce qu'il dit et ne dit pas, ce qu'il en dira et ce qu'il sous-entend, voire ce qu'il rédige comme porte-plume, car l'une des leçons de l'ascèse archivistique qu'ont pu mener, parmi d'autres, Victor Del Litto ou Elaine Williamson, est de faire ressortir les compétences de rédacteur de Beyle, et l'estime que lui valait sa plume administrative ${ }^{5}$.

5 Les historiens de Napoléon savent au moins une chose de Stendhal : il ne faut pas en parler à la légère sous peine d'en être la dupe. Dans ces conditions, l'historien de la période ne peut guère enrichir qu'à la marge le patrimoine des écrits de Stendhal par les documents le mentionnant croisés au cours de ses dépouillements, à l'instar de cet 
état des auditeurs parlant des langues étrangères, et dans lequel Beyle, « inspecteur du Garde meuble ", apparaît dans la colonne des italophones, aux côtés de 103 collègues parlant allemand, anglais, espagnol ou hollandais ${ }^{6}$.

6 Conforme en cela au tropisme de la période, c'est essentiellement sur le rapport de Stendhal à Napoléon que se succèdent les travaux. D'ailleurs, l'histoire sociale n'est-elle pas vouée à buter sur Stendhal, c'est-à-dire sur cet individu réfractaire à la double opération de réduction et de montée en généralité comme type, lui qui n'a cessé de se démarquer tout en s'employant à typifier ses contemporains? Stendhal, ce républicain rouge et noir ${ }^{7}$, ne sert pas même de référence commune aux travaux qui, au fil des années de Bicentenaire, ont fluidifié le cap entre la Révolution et le régime napoléonien ${ }^{8}$. Il est vrai que la dissimulation à laquelle recourt Stendhal dans ses journaux restreint les jugements directs sur les faits politiques dont sont friandes les anthologies.

7 Proprement atypique, Stendhal ne fournit donc qu'un contre-exemple, un regard critique propre à démasquer le conformisme de ses contemporains, à moins de manier la clef générationnelle et de souligner dès lors l'ouverture des possibles, tant politiques que sociaux en termes de carrières, et moraux (comme le divorce), qui s'offrent à ceux ayant grandi avec la Révolution, à la faveur de la péremption des normes anciennes. Si ce mode de mise en contexte est déjà utilisé pour situer Henri Beyle ${ }^{9}$, il manque sans doute encore une formalisation prosopographique pour mieux jauger sa trajectoire à l'aune des groupes et corps auxquels il a appartenu (les élèves de l'école centrale de Grenoble, les commissaires de guerre, les auditeurs au Conseil d'État).

\section{Stendhal n'est pas un collègue}

8 Témoin retors et irréductible pour les historiens, Stendhal n'est pas non plus un collègue, pour faire écho au Thucydide de Jacqueline de Romilly repris par Pierre Karila-Cohen à propos des préfets des monarchies postrévolutionnaires ${ }^{10}$. En effet, la sagacité des éclairages qu'il offre dans ses œuvres historiques ne doit pas pour autant les faire admettre comme des analyses que l'on placerait sur le même plan distancié, hors de l'histoire, que celles d'un historien d'aujourd'hui. De fait, même un lecteur aussi pénétré que Patrice Gueniffey des approches menées par les historiens libéraux pour mieux cerner Bonaparte ne s'appuie guère sur Stendhal, sauf comme témoin ou comme écrivain ${ }^{11}$. La part d'emprunts dans les textes historiques de Stendhal pourrait d'ailleurs conduire à en dévaluer l'intérêt. Il y a pourtant une leçon essentielle à tirer et à méditer de la Vie de Napoléon: l'inadéquation entre le titre, le projet et le résultat final que pointait le Milanais Pietro Borsieri ${ }^{12}$, est une vraie aporie, puisque l'homme Napoléon s'efface dès 1796 derrière son rôle historique. Or même les meilleurs historiens-biographes actuels de Napoléon ne la peuvent surmonter, et pour cause, si bien que les réussites et les limites d'un travail comme celui, dernièrement, de Natalie Petiteau sont en définitive très stendhaliennes ${ }^{13}$. Mais au-delà de ce paradoxe du biographe sur lequel Stendhal a buté, son œuvre est riche en mots qu'il jette comme par boutade ou par inadvertance, et qui se révèlent d'une grande acuité historique :

Le premier consul se convainquit bien que la vanité était en France la Passion nationale. Pour satisfaire à la fois cette passion de tous et sa propre ambition, il fut attentif à agrandir la France et à augmenter son influence en Europe. Le Parisien en trouvant un matin dans son Moniteur un décret commençant par ces mots : La Hollande est réunie à l'Empire, admirait la puissance de la France, voyait Napoléon 
bien supérieur à Louis XIV, se faisait gloire d'obéir à un tel maître, oubliait qu'il avait été vexé, la veille, par la conscription ou les Droits Réunis, et songeait à demander pour son fils une place en Hollande.

À l'époque dont nous parlons, le Piémont, les États de Parme et l'île d'Elbe furent successivement annexés à la République. Ces réunions partielles fournissaient à la conversation $^{14}$.

Sous une gangue assez banale, le propos invite à dynamiser les études napoléoniennes en réfléchissant à ce que pourrait être une New Imperial History, c'est-à-dire ce tournant qui a transformé la manière d'aborder l'Empire britannique. Dans les mots de Stendhal, on retrouve non seulement les prémisses d'une propagande impériale manipulatrice telle que l'avait pointée John Mackenzie mais encore l'idée que l'expansion impériale modifie les cadres de pensée métropolitains, que l'Empire s'insinue dans la vie de tout un chacun, crée des débouchés et un imaginaire, nourrit les conversations ou les correspondances ; bref, que pour la France napoléonienne aussi l'Empire est à étudier at hom $e^{15}$. De là aussi l'enquête prosopographique à mener sur le marché des emplois impériaux ${ }^{16}$. C'est un lourd investissement mais Stendhal est un guide sûr en matière de patronage bureaucratique. Auditeur au Conseil d'État attaché à la Liste civile et affecté à l'Intendance générale de la Maison de l'Empereur, ne dirigeait-il pas le bureau chargé des affaires de la Hollande? Il était donc aux premières loges pour recevoir pareilles demandes de place dont on trouve de fait, pour chaque ministère, tout un lot à chaque nouvelle annexion.

C'est donc parce qu'il émane d'un homme maîtrisant les rouages impériaux pour avoir contribué à les faire fonctionner que ce genre de passage est susceptible d'éclairer l'historien ; cela signifie par conséquent que l'avis des homologues de Stendhal, placés dans les mêmes situations de responsabilité, promettent d'être tout aussi utiles. On rejoint ainsi la démarche ordinaire de l'historien qui, face à un émetteur de sources, en évalue la fiabilité au vu de la sphère de connaissances (in)directes que lui ouvrait sa trajectoire biographique; d'où les notices introductives au seuil des Mémoires qui ont fait l'objet d'une édition critique. Lorsque l'auteur prétend cependant faire œuvre d'histoire et refuse de s'en tenir à l'auto-production des sources de ses analyses, d'autres filtres sont à intercaler: ce qu'écrit Stendhal et ses contemporains en dit moins sur eux-mêmes que sur leur conception de l'histoire.

\section{À quoi sert l'histoire pour Stendhal et ses pairs ?}

11 Leur familiarité avec l'histoire tient à sa valeur formatrice. Décidé à se donner les moyens de faire une carrière publique, Stendhal se fixe, le 14 mars 1806, le programme suivant :

Me chercher moi-même, aller beaucoup en société, acquérir des talents pour le monde ou pour le travail avec $\mathrm{D}[\mathrm{aru}]$, s'il m'appelle. J'ai le plus grand besoin d'un plan d'instruction. Il faut absolument que j'apprenne l'histoire, que je revoie la géographie, pour n'être pas trop étranger aux idées de politique si j'ai besoin d'en prendre ${ }^{17}$.

12 Ce plan de formation, dont le détail apparaît au fil des lectures qu'il conseillait à sa sœur Pauline, s'applique à d'autres jeunes gens soucieux d'actualiser leurs savoirs géographiques dans une Europe en mouvement ${ }^{18}$, d'acquérir l'aplomb pour se tenir prêts, à la hauteur des circonstances et des places qu'ils convoitent ${ }^{19}$. Il existe en effet une certaine demande sociale d'histoire, que les lycées napoléoniens, où dominent les 
mathématiques et le latin, ont prise en compte ${ }^{20}$. En dehors de l'institution scolaire, l'apprentissage s'opère par des notes prises à la suite de tel ou tel ouvrage, ou au fil de lectures partagées avec des proches, à la façon dont Stendhal et Crozet travaillaient l'histoire de la Révolution en 1810. Cette discipline leur apprend à juger des faits dont on s'est enquis au préalable de l'authenticité.

13 Sans doute la Révolution a-t-elle fait basculer Stendhal et ses contemporains dans un nouveau régime d'historicité : le caractère unique de l'événement leur font prendre leurs distances avec la notion d'exemplum ${ }^{21}$, si bien que Stendhal n'a " pas voulu faire de ces Mémoires un cours de connaissance du cœur humain ${ }^{22} »$. Cependant, la conception d'une histoire comme réservoir de leçons universelles n'est pas totalement obsolète : l'histoire reste un savoir utile sur les hommes et la politique; en ces temps de nouvelle République, l'histoire romaine demeure une source d'inspiration. Le pas est vite franchi d'une histoire qui donne des clefs de compréhension de la politique à une histoire qui agit sur la politique. Ainsi le républicain de 1801 appelait-il de ses vœux une histoire romaine qui réveillerait les consciences ${ }^{23}$; ainsi le fonctionnaire impérial recommandet-il, dix ans plus tard, le choix d'historiographes officiels, en songeant peut-être à luimême, pour gagner durablement à Napoléon le cœur des Français ${ }^{24}$.

Cette utilité de l'histoire est toutefois moins prospective que réparatrice. C'est par l'écriture que l'auditeur déchu se retrouve par lui-même une place dans l'histoire tout comme le recul de l'histoire rend justice à la domination napoléonienne en Italie (Rome, Naples et Florence, 1817/1826). Si la leçon est dure à faire passer auprès de ses contemporains, pris dans les enjeux du court terme, sensibles aux effets de mode et intéressés à la vulgate momentanément dominante, l'histoire offre à terme une chance aux approches à contre-courant. Cette conception conduit Stendhal à en faire un instrument de rectification des fautes commises : à grands renforts de formules telles que «Il aurait fallu», Stendhal se prend à conseiller Napoléon dans la Vie qu'il lui consacre, pour déchirer l'écran courtisan qui a altéré ses choix, et pour mettre son énergie au service du bien commun. Or il ne faudrait être que le lecteur d'un seul auteur pour ne pas repérer que de tels usages se retrouvent ailleurs. Au fond, qu'y a-t-il de proprement stendhalien dans le Stendhal historien?

Encore faut-il passer outre quelques habitudes. Il est sinon de bon ton du moins courant de signaler Stendhal comme une anomalie parmi les juristes et légistes du Conseil d'État. Embarrassé par la place à donner aux fortes personnalités dans un corps, le dictionnaire biographique qui leur est dédié considère Stendhal avec un amusement fatigué : celui-ci a le tort d'usurper la notoriété qui devrait revenir à des collègues obscurs mais dévoués ${ }^{25}$. Or n'est-ce pas le lieu de rappeler que Pierre Daru lui-même a mis à profit sa retraite pour s'adonner à l'écriture de l'histoire, celle de la Bretagne et surtout celle de Venise? Les écrivains et diplomates n'ont-ils pas leur périmètre historiographique ${ }^{26}$ ? Stendhal y incarne d'ailleurs la figure la plus nette du diplomate écrivain ${ }^{27}$. On sait à quel point l'exercice d'un «second métier » est structurel pour les écrivains d'hier et d'aujourd'hui, et c'est là, alors, aussi une " condition historienne ${ }^{28}$ ". Les stendhaliens ayant gagné le procès en désinvolture intenté à l'auditeur Beyle, il reste à faire l'autre bout du chemin en mettant en évidence sinon le dilettantisme de ses collègues, du moins leurs pratiques d'écriture ${ }^{29}$ : la singularité de Stendhal s'estompe dès lors que l'on cite les œuvres de Lamothe-Langon, de Prévost d'Arlincourt ou d'Alexandre Soumet. Sans doute ne forment-ils qu'une minorité, mais cette présence 
n'a rien de surprenant si l'on veut bien se souvenir que le Conseil d'État est une pépinière d'administrateurs ouverte à des jeunes gens encore en quête d'eux-mêmes.

On ne s'illusionnera pas toutefois sur l'utilité d'un décompte des historiens au Conseil d'État. D'une part, on ne connaît guère de ces fonctionnaires que leurs ouvrages dûment publiés; à ce titre, Stendhal ne figurerait pas dans cette liste faute d'avoir achevé et édité ses entreprises historiques. D'autre part, le partage des genres entre Mémoires et Histoire est trop poreux. Ce qui ressort, c'est la diversité des écritures historiques possibles, aboutissant à une typologie ramifiée, dont on écartera les Souvenirs stricto sensu, rédigés pour l'instruction des siens, sans visée éditoriale ni prétention à l'histoire ${ }^{30}$ et les monographies historiques réalisées dans le sillage de fonctions officielles ${ }^{31}$. De même, on laissera de côté les œuvres de conseillers d'État dont les expériences, du fait du décalage générationnel, sont bien éloignées de Stendhal, parce que trop âgés (Andréossy, Berlier, Lameth, Nougarède ou Roederer), ou, plus largement, le travail de trop jeunes historiens : on songe à Alexandre Barginet, un Grenoblois né 1797, qui s'est jeté à corps perdu au service des Cent-Jours, avant de prendre la plume pour défendre le bilan de Napoléon ${ }^{32}$. Le parallèle est en effet plus opératoire lorsqu'avec Barante, on tient un auditeur de l'âge de Beyle, passé par la gestion des pays conquis, lorsqu'avec le Grenoblois Joseph Rey, il y a matière à envisager des trajectoires socio-politiques saillantes, ou lorsqu'avec Dubois-Aymé, on peut s'appuyer sur de nombreux manuscrits, également conservés à Grenoble ${ }^{33}$ : avant d'être historiens, ces hommes ne sont-ils pas les archivistes d'eux-mêmes ${ }^{34}$ ? Les esquisses de Dubois-Aymé révèlent un travail d'historien presque invisible au niveau de ses publications ${ }^{35}$.

\section{Quelle écriture pour l'histoire?}

17 Les distinctions auxquelles poussent les parcours biographiques ne sont du reste qu'un paramètre possible. Au sein de ces textes, se dessine un autre clivage qui tient aux choix d'écriture. Le style adopté se rapporte à la délimitation du sujet tant il s'avère que l'on ne s'exprime pas de la même façon lorsqu'il s'agit de raconter des épisodes des $\mathrm{XVI}^{\mathrm{e}}$ et $\mathrm{XVII}^{\mathrm{e}}$ siècles ou lorsque l'on traite du passé immédiat - les qualités de coloriste sont alors inutiles et suspectes. Pour le général Foy, en quête de modèles pour guider sa propre écriture historique - il entend reconstituer toutes les opérations dans la péninsule ibérique, et pas seulement raconter sa guerre d'Espagne -, un auteur comme Barante a la plume trop élégante, polie, plaisante pour tout dire, pour être honnête, sérieuse et véridique ${ }^{36}$. Bien que ce dernier, éminent représentant de l'école narrative, défende l'authenticité des soubassements historiques de son œuvre, il fait figure, au même titre que Daru, que le diplomate-sénateur Pradt ou que l'ingénieur-préfet SainteAulaire, d'historien de rencontre comparé à « la jeune génération des Thiers et des Mignet ${ }^{37}$ ».

Stendhal et ses pairs aspirent à un style sobre et exact ${ }^{38}$. Simple et positive, parce que porteuse de connaissances avérées que l'on entend transmettre à d'autres, l'écriture de l'histoire n'autorise pas les approximations que rejette Stendhal derrière le style brumeux et flottant de Chateaubriand. L'exigence est cependant difficile à atteindre faute de trouver le ton juste, d'obtenir en tant qu'auteurs ce qu'ils cherchent comme lecteurs. De fait, l'histoire que ces hommes entreprennent de rédiger est aussi celle qu'ils aimeraient lire; en cela, les questions que pose leur production sont celles 
de leur propre réception des œuvres historiques: «J'écris cette histoire telle que j'aurais voulu la trouver écrite par un autre ${ }^{39}$. »

19 L'histoire-récit que composent Stendhal et ses pairs est bien différente des grands systèmes dispensés dans les écoles centrales de la République sur le modèle des leçons à l'École normale supérieure ${ }^{40}$. Il n'y a guère que Portalis fils, maître des requêtes au Conseil d'État à avoir bâti une philosophie de l'histoire ${ }^{41}$. Pour les autres, parce que l'histoire se doit d'être véridique, les témoins ont pour l'écrire l'inégalable atout de l'authenticité. «Il y était » : tel est l'argument décisif par lequel le général Foy soupèse ses lectures, dresse une hiérarchie complaisante à l'égard des témoins forts de leur vécu, et dédaigneuse envers les historiens de cabinet ${ }^{42}$. Né la même année que Stendhal, Eugène Labaume, qui, originaire du Vivarais, a fait carrière aux côtés du prince Eugène dans le royaume d'Italie comme ingénieur-cartographe, avait commencé sous l'Empire une œuvre d'historien encore maladroite et matinée de plagiat (Histoire abrégée de la république de Venise, 1811), avant de dominer momentanément la jeune historiographie napoléonienne en livrant dès 1814 une Relation circonstanciée, devenue complète lors de sa réédition, de la campagne de Russie puis une Histoire de la chute de l'Empire de Napoléon (1820). Ses œuvres relèvent à la fois des Mémoires, de la synthèse historique et de l'essai politique, et sont portées par un esprit critique que ne renierait pas Stendhal. Mieux, en 1818, Labaume théorise, pour la défendre, sa pratique historienne dans ses Réflexions sur les inconvénients et les avantages de l'histoire contemporaine, par lesquelles il entend démontrer l'apport irremplaçable des témoignages de ceux qui ont pris une part active aux événements. C'est là exprimer le goût d'une génération à peine retirée des affaires et qui n'admet pas que l'histoire de l'Empire, leur histoire en vérité, puisse se faire sans eux.

20 Au fond, parler de Stendhal historien quand on est soi-même historien revient à parler de soi, de sa conception de sa discipline et de sa pratique des sources. N'était-il pas essentiel d'introduire ce regard historien si peu présent lorsqu'il s'agit de Stendhal? Que peut donc alors apporter l'historien? Assurément, il peut opérer un mouvement de décentrement salutaire, via une mise en contexte et en situation. Il n'est d'ailleurs guère besoin de beaucoup s'éloigner du cercle de relations de Stendhal pour disposer de l'effet de perspective utile car l'ancien auditeur écrit non seulement pour ses pairs ( J'écris pour des gens qui ont lu Montesquieu ou qui ont eu l'occasion de donner dans leur vie 2000 signatures officielles $\left.{ }^{43} »\right)$ mais encore comme ses pairs, s'adonnant comme lui à toute une gamme d'écritures personnelles qu'ils feront fructifier par la suite. Ces hommes retrouveront en effet dans leurs ego-documents des matériaux pour l'histoire vécue qu'ils ambitionnent. Conscients toutefois qu'il leur manque encore quelque chose, ils vont chercher de préférence l'histoire dans les Mémoires, et, écartelés par un tel rapport au temps et à l'écriture, ils s'exposent dès lors comme Stendhal à tâtonner dans leurs propres travaux historiques. 


\section{NOTES}

1. Stendhal, Napoléon. Vie de Napoléon. Mémoires sur Napoléon, édité par C. Mariette, Paris, Stock, 1998, chap. 1, p. 15.

2. I. Jablonka, L'Histoire est une littérature contemporaine. Manifeste pour les sciences sociales, Paris, Le Seuil, 2014.

3. N. Petiteau, Écrire la mémoire. Les mémorialistes de la Révolution et de l'Empire, Paris, Les Indes Savantes, 2012.

4. F. Michel, Fichier stendhalien, Genève, Slatkine, 2003 [1964], 2128 p.

5. E. Williamson, "Stendhal sous l'Empire", dans Stendhal, Correspondance générale, édité par V. Del Litto, Paris, Champion, 1997, t. II, p. IX-XIX ; « Beyle-Stendhal en 1809 : les énigmes de la Campagne de Vienne", dans M.-R. Corredor (éd.), Stendhal, Vienne, l'Autriche, Recherches \& Travaux, $\mathrm{n}^{\circ} 79$, Grenoble, Ellug, 2011, p. 35-71.

6. État des auditeurs parlant des langues étrangères, s.d. [été 1791], Archives nationales, $\mathrm{F}^{7} 6346$.

7. Pour reprendre le titre de l'exposition qui a eu lieu dans l'appartement Gagnon-Musée Stendhal, de mai 2015 à mai 2016.

8. Les héritages républicains sous le Consulat et l'Empire, édité par J.-O. Boudon et P. Bourdin, Annales historiques de la Révolution française, $\mathrm{n}^{\circ}$ 346, 2006-1.

9. «Ainsi, à 18 ans, le futur romancier est un excellent spécimen de la génération née à la veille de la Révolution : il hait les tyrans, il croit sincèrement à la vertu républicaine », V. Del Litto, "Stendhal et Napoléon », Une somme stendhalienne. Études et documents 1935-2000, Paris, Champion, 2002, p. 867.

10. P. Karila-Cohen, « Les préfets ne sont pas des collègues. Retour sur une enquête ", Genèses, $\mathrm{n}^{\circ}$ 79, 2010/2, p. 116-134.

11. P. Gueniffey, Bonaparte, Paris, Gallimard, 2013.

12. Son jugement sur la Vie de Napoléon est reproduit en appendice de l'édition citée de C. Mariette (p. 200).

13. «Il est hélas impossible de savoir comment Bonaparte accueillait au jour le jour ces différentes nouvelles, et finalement seuls ses actes, durant le Consulat et l'Empire, traduisent ce qu'il est et ce qu'il pense", N. Petiteau, Napoléon. La nation incarnée, Paris, Armand Colin, 2015 , p. 124.

14. Stendhal, Vie de Napoléon, ouvr. cité, p. 50.

15. A. Lignereux, L'Empire des Français, 1799-1815, t. 1 de La France contemporaine, Paris, Le Seuil, coll. « Points Histoire », 2014, p. 259-284.

16. A. Lignereux, Les "Impériaux», de l'Europe française à la France postnapoléonienne (1 ìre moitié du $\mathrm{XIX}^{e}$ siècle), projet de recherche retenu par l'Institut universitaire de France (2016-2021).

17. Stendhal, Journal, édité par H. Martineau revue par X. Bourdenet, Paris, Gallimard, 2010, p. 436.

18. A. Lignereux, «La guerre, ça sert, d'abord, à faire de la géographie: les Français face à l'Europe de Napoléon ", La géographie à l'époque napoléonienne, dossier dirigé par N. Todorov, Revue de Géographie historique, $\quad \mathrm{n}^{\circ} 5, \quad<\mathrm{http}$ ://rgh.univ-lorraine.fr/articles/view/49/ La_guerre_ca_sert_d_abord_a_faire_de_la_geographie>

19. «Je refis mon histoire en dressant un tableau synoptique général », se souvient Barthélémy. À vingt ans, il travaillait à la préfecture de la Roër et bénéficiait de l'intérêt du préfet Lameth, qui lui avait tracé un plan de formation (Souvenirs d'un ancien préfet, 1787-1848, Paris, Dentu, 1885, p. 38). 
20. A. Bruter, "L'enseignement de l'histoire dans les lycées napoléoniens ", dans J.-O. Boudon (éd.), Napoléon et les lycées. Enseignement et société en Europe au début du XIXe siècle, Paris, Nouveau Monde éditions - Fondation Napoléon, 2004, p. 99-114.

21. Journaux \& Papiers Stendhal. Volume I -1797-1804, édition établie par C. Meynard, H. de Jacquelot, M.-R. Corredor, Grenoble, Ellug, 2013, p. 8.

22. Stendhal, Mémoires sur Napoléon, ouvr. cité, $1^{\mathrm{er}}$ projet préface, p. 250.

23. 11 f[ructid]or 11 [30/08/1801], Stendhal, Journaux \& Papiers, ouvr. cité, p. 366.

24. 8 juin 1811, Journal, ouvr. cité, p. 752 ; Sur cette histoire mise au service de l'Empereur, voir J. K. Burton, Napoleon and Clio: historical writing, teaching and thinking during the First Empire, Durham, Carolina academic press, 1979.

25. R. Drago, J. Imbert, J. Tulard et F. Monnier (éd.), Dictionnaire biographique des membres du Conseil d'État, 1799-2002, Paris, Fayard, 2004, p. III.

26. L. Badel, G. Ferragu, S. Jeannesson et R. Metz (éd.), Écrivains et diplomates. L'invention d'une tradition, $\mathrm{XIX}^{e}-\mathrm{XXI}{ }^{e}$ siècles, Paris, Armand Colin, 2012.

27. «Introduction » et Y. Bruley, «Le diplomate français au XIX ${ }^{\mathrm{e}}$ siècle, entre belles-lettres et littérature ", Ibid., p. 22 et 46-48.

28. B. Lahire, La condition littéraire. La double vie des écrivains, Paris, La Découverte, 2006.

29. J.-N. Pascal (éd.), Quand les premiers préfets courtisaient la Muse, numéro thématique des Cahiers Roucher-André Chénier, $\mathrm{n}^{\circ}$ 27, 2008.

30. Comme les Souvenirs du duc de Broglie (1785-1870): "J'écris mon histoire, je n'écris ni l'histoire, ni pour l'histoire ", Paris, Calmann-Lévy, 1886, p. 206.

31. À l'instar des travaux de son compatriote H. Berriat (Coup d'œil historique sur les anciens corps du commissariat des guerres et de l'inspection aux revues, Grenoble, Baratier, 1832).

32. A. Barginet, Sur Napoléon, ou Réponse aux journaux contre-révolutionnaires qui s'intitulent "Quotidienne ", "Gazette de France », "Journal des débats » et " Drapeau blanc ", Paris, marchands de nouveautés, 1821.

33. On y trouve notamment une Histoire de la campagne d'Égypte et de Syrie, Bibliothèque municipale de Grenoble, Rés. 8320.

34. Le général Foy tenait ainsi un journal à chaud de ses campagnes, voir J.-C. Caron, Les deux vies du général Foy (1775-1825). Guerrier et législateur, Seyssel, Champ Vallon, 2014, "Un archiviste de soi », p. 26.

35. Rectification relative aux Mémoires de M. de Bourrienne au sujet de la prise de Malte, écrite par Dubois-Aymé, Toulon, Aurel, 1830.

36. J.-C. Caron, ouvr. cité, p. 274.

37. Ibid., p. 37 et 279.

38. Stendhal, Mémoires sur Napoléon, ouvr. cité, $2^{\mathrm{e}}$ projet de préface, p. 252.

39. Ibid., $1^{\mathrm{er}}$ projet de préface, p. 249.

40. L'École normale de l'an III. vol.2. Leçons d'histoire, de géographie, d'économie politique, Paris, Éditions de la rue d'Ulm, 2012.

41. J. Portalis, Du devoir de l'historien de bien considérer le caractère et le génie de chaque siècle, Paris, Bernard, an VIII ; De la Guerre dans ses rapports avec les destinées du genre humain, Orléans, ColasGardin, 1856.

42. J.-C. Caron, ouvr. cité, p. 274. C'est précisément la justification des mémorialistes : «En un mot, et ce sera tout mon pauvre mérite, je dirai :j'étais là, telle chose m'advint ", Souvenirs du feu duc de Broglie, 1785-1870, Paris, Calmann-Lévy, 1886, p. VII [je souligne].

43. Stendhal, Mémoires sur Napoléon, ouvr. cité, $1^{\mathrm{er}}$ projet préface, p. 250. 


\section{RÉSUMÉS}

Ils sont nés autour de 1780 ; hauts fonctionnaires, ils ont servi Napoléon dans l'Europe conquise, puis ont pris leurs distances avec l'empereur au moment de sa chute avant de suivre des parcours contrastés. Plusieurs ont connu une carrière préfectorale, quelques-uns ont été nommés ministres; Henri Beyle est devenu Stendhal. Tous cependant ont écrit des Mémoires ou des Souvenirs, des essais et des monographies à caractère historique, des histoires même. Les formes sont diverses, les genres hésitants ; quelques œuvres ont été publiées de leur vivant, beaucoup à titre posthume ; certaines sont restées à l'état d'archives. Et c'est précisément cette variété qui est susceptible d'éclairer sous un jour nouveau les tentatives de Stendhal, car ses incursions en histoire ont été aussi celles de ses pairs.

They are born around 1780; senior officials, they have served Napoleon in conquered Europe, then, they have distanced themselves from the Napoleonic power at the time of the imperial collapse and they have found their own way. Many of them have had prefectural careers, some have been promoted to ministers; Beyle has become Stendhal. However, all of them have written Memoirs, historical essays or monographs, and even history books. This takes several different forms; the genres are hesitants; some works were published before their death; a lot released posthumously; few of these manuscripts remained in draft manuscript form. And that is precisely what can bring a new angle to the Stendhal's attempts, because his incursions into the history field were also experienced by his peers.

\section{AUTEUR}

\section{AURÉLIEN LIGNEREUX}

Institut d'Études Politiques de Grenoble

LARHRA UMR 5190

Aurélien Lignereux est maître de conférences en histoire contemporaine à l'IEP de Grenoble et membre de l'Institut Universitaire de France (Junior, 2016) et du LARHRA (UMR CNRS 5190). Spécialiste des forces de l'ordre et des révoltes (La France rébellionnaire. Les résistances à la gendarmerie, 1800-1859, PUR, 2008 ; Servir Napoléon. Policiers et gendarmes dans les départements annexés, 1796-1814, Champ Vallon, 2012 ; Chouans et Vendéens contre l'Empire. 1815 : l'autre guerre des Cent-Jours, Vendémiaire, 2015), il centre désormais ses recherches sur l'expatriation des fonctionnaires dans l'Europe napoléonienne et la valorisation de leur capital impérial dans la France d'après 1815 (L'Empire des Français, 1799-1815, Le Seuil, 2012 ; avec Marie-Bénédicte Vincent, Réintégrer les fonctionnaires. «L'après-épuration » en Europe, $\mathrm{XIX}{ }^{e}-\mathrm{XX}{ }^{e}$ siècle, revue Histoire \& Mesure, vol. XXIX, 2014-2). 